\title{
Customizable nanotweezers for manipulation of free-standing nanostructures
}

Bøggild, Peter; Hansen, Torben Mikael; Mølhave, Kristian; Hyldgård, Anders; Jensen, M. O.; Richter, Jacob; Montelius, Lars; Grey, Francois

\section{Published in:}

Proceedings of The 2001 1st IEEE Conference on Nanotechnology

Link to article, DOI:

10.1109/NANO.2001.966399

Publication date:

2001

Document Version

Publisher's PDF, also known as Version of record

Link back to DTU Orbit

Citation (APA):

Bøggild, P., Hansen, T. M., Mølhave, K., Hyldgård, A., Jensen, M. O., Richter, J., Montelius, L., \& Grey, F. (2001). Customizable nanotweezers for manipulation of free-standing nanostructures. In Proceedings of The 2001 1st IEEE Conference on Nanotechnology (pp. 87-92). IEEE. https://doi.org/10.1109/NANO.2001.966399

\section{General rights}

Copyright and moral rights for the publications made accessible in the public portal are retained by the authors and/or other copyright owners and it is a condition of accessing publications that users recognise and abide by the legal requirements associated with these rights.

- Users may download and print one copy of any publication from the public portal for the purpose of private study or research.

- You may not further distribute the material or use it for any profit-making activity or commercial gain

- You may freely distribute the URL identifying the publication in the public portal 


\title{
Customizable nanotweezers for manipulation of free-standing nanostructures
}

\author{
P. Bøggild, T. M. Hansen, K. Mølhave, A. Hyldgård, M. O. Jensen, J. Richter, L. Montelius*, F. Grey \\ Mikroelektronik Centret \\ Technical University of Denmark, Building 345 east, 2800 Lyngby, Denmark \\ *Div of Solid State Physics \& the Nanometer Consortium, Lund Universit, Box 118, 221 00 Lund. Sweden \\ Email: $p b @ m i c . d t u . d k$
}

\begin{abstract}
We present a novel nanotweezer device for manipulation and measurement of free-standing nanostructures, where the shape of the tweezer tips can be customized for the application. Electrostatic actuators with submicron interelectrode spacings are fabricated on a batch level using silicon microfabrication techniques. The actuators are capable of opening and closing with respect to the neutral position, and the full range of actuation exceeds $330 \mathrm{~nm}$. The nanotweezer tips are fabricated using electron beam induced deposition; an electron beam of a scanning electron microscope is focused at the ends of the cantilevers, leading to the formation of $40-100$ $\mathrm{nm}$ diameter carbon-based tips. Careful alignment of the substrate and the beam, as well as proper selection of growth mode, results in converging tips forming nanogaps down to $20 \mathrm{~nm}$. The nanotweezers can be metallized to be used for electrical measurements of nanostructures.
\end{abstract}

\section{Introduction}

The field of nanomanipulation was pioneered by Eigler [1] and others, who showed that single atoms could be precisely positioned using the sharp tip of a scanning tunneling microscope. Scanning probe microscopes have since then been used for a range of remarkable nanomanipulation applications, including fabrication of room temperature quantum devices [2], switching of single hydrogen atoms on a $\mathrm{H}$-passivated silicon surface [3] and investigations of frictional and mechanical properties of nanostructures using a realtime force feedback system [4,5]. Nanotechnology research has now established that carbon and silicon nanowires exhibit promising mechanical and electrical properties to be used as active parts of novel sensors and transducers [6-8]. For electronics, nanowires could both function as the active devices and as interconnects accessing the devices [8].

One great challenge is to position such small objects quickly, precisely and reliably, for instance on a set of electrodes. An intriguing scenario for device fabrication with just a few active nanosize components is to pick and place the components one by one using a suitable manipulation device. Scanning probe microscopy has been quite successful in manipulating carbon nanotubes $[4,6,7]$ and silicon nanowires [8] already deposited on a surface, whereas manipulation of free-standing nanoscale objects is a different matter.

One strategy is simply to exploit the van der Waals forces to break off and lift a single wire from a tangle of wires or from a surface, such as described in Ref. 7 .

The alternative approach, investigated in this study, is to use opposing forces applied by a form of tweezers. Kim and Lieber [9] first demonstrated grabbing of nanoparticles and wires using two carbon nanotubes, where the actuation was accomplished by applying an electrostatic potential between the nanotubes. However, such a voltage difference might create a considerable electrical field at the tweezer tips, which in turn can lead to undesired changes in the behavior of the studied object. Kakushima et al. [10] recently presented batch-fabricated nanotweezers with thermal expansion actuators, thus avoiding this problem.

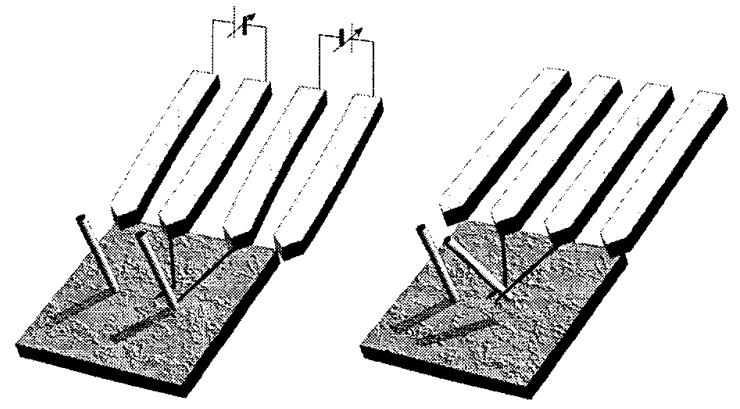

Figure 1: Nanotweezers grabbing a free-standing nanowire. Left: The nanotweezers are opened by applying voltages between the inner and outer electrodes, leaving the inner electrodes at the same potential. Right: The voltage is decreased in order to close the tweezers.

We present here a nanotweezer device combining a batch-fabricated electrostatic microactuator with addon customizable nanotips. The microactuator is designed to avoid applying an electrical voltage between the tweezer tips (see Fig. 1). This makes the device suitable for handling fragile structures. The use 
of electron beam induced deposition (EBD) provides free-form 3D nanostructuring, ensuring a considerable flexibility in terms of the shape and dimensions. Using this approach, tweezer gaps down to $20 \mathrm{~nm}$ have been fabricated in a straightforward fashion. The EBD technique has been known for more than a decade, and has primarily been used for high-aspect ratio SPM tips [11], and related devices such as high-brightness electron emitters [12] and nano four-point conductivity probes [13].

\section{Fabrication of nanotweezers}

The microactuator consists of an array of metalcoated silicon oxide microcantilevers extending over the edge of a silicon support. Several electrode layouts have been implemented with up to five electrodes. In all cases, EBD tips have been grown on two gripper electrodes (Fig. 2a), while the driver electrodes (Fig. 2b) are used to set up an electrostatic field and thereby deflect the gripper electrodes.

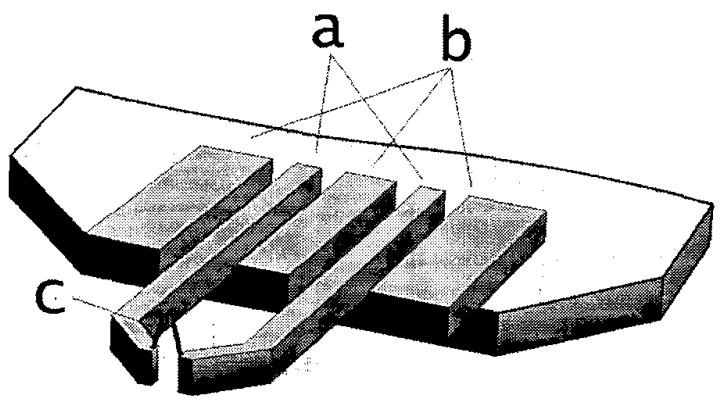

Figure 2. Illustration of five-electrode actuator. The two gripper cantilevers (a) can be made to deflect inwards as well as outwards, by applying voltages on the driver electrodes (b) while keeping both gripper electrodes at the same potential. A nanotip (c) is deposited on each gripper electrode.

\subsection{Batch fabrication of the microactuator}

The silicon support structure was fabricated in a simple, conventional silicon-processing technique. A $1 \mu \mathrm{m}$ thick layer of thermal oxide was grown on both sides of a $\mathrm{Si}(100)$ wafer. Using UV-lithography, the cantilever electrode pattern was transferred to the wafer front surface, and defined by reactive ion etching (RIE) using a chromium lift-off mask. A thin low-stress nitride membrane was deposited on both sides and opened at the backside. The cantilevers were then released in a $\mathrm{KOH}$ etch. After removal of the nitride membrane, an isotropic etch undercuts the cantilevers.

The undercut structure allows the silicon support chips to be coated with a thin metallic layer, typically $100 \AA \mathrm{Ti}$ and $800 \AA \mathrm{Au}$, individually or on a full wafer basis, without short-circuiting the cantilevers. The thickness and composition of the metallic layer can be selected freely, since the metallization is done as the final processing step. The microfabrication process is described in more detail in Ref. 14.

The initial tests were done with four-electrode layouts (see Fig. 1), while later being replaced by the more versatile five-electrode layout (see Fig. 2). A similar electrode layout has earlier been implemented as a microgripper by $\mathrm{Kim}$ et al. [15].

\subsection{EBD fabrication of nanotweezer tips}

We used a JEOL 6430F field emission microscope operating at a base pressure of $10^{-5}$ Torr. By focusing the electron beam of a scanning electron microscope on the ends of the microcantilevers, hydrocarbon molecules present in small concentrations near the beam spot are cracked. This leads to the formation of a thin tip composed of carbon residues, growing in the direction of the electron beam.

The diameter is typically in the $50-200 \mathrm{~nm}$ range depending on the growth mode (see section 2.3) and the growth parameters. The length can become tens of microns, simply by continuation of the growth process. During growth, the supporting electrodes are maintained at the ground potential with respect to the scanning electron microscope. This provides a drain for the electron beam and thus reduction of charge buildup. In order to make the nanotips conducting, a second metallization ( $100 \AA \mathrm{Ti} / 600 \AA \mathrm{Au}$ ) step can be done.

\subsection{Growth modes}

Three different growth modes for making converging supertips are used: vertical growth, where the electron beam is parallel to the tip, lateral growth where the beam is perpendicular to the tip, and flood growth where the beam is scanning during growth, thus inducing a uniform deposition over a rectangular area. These three modes are illustrated in Fig. 3.

With vertical growth. the microactuator had to be tilted and rotated with respect to the beam, so that the nanotips grew in converging directions. It is however easy to ensure that the tips grow in the same plane. For each step the growth rate is measured from the SEM images. The tips are grown in an alternating, iterative fashion, using successively shorter deposition times, until the gap size is about 100-300 $\mathrm{nm}$. With this growth mode it becomes difficult to reduce the gap further, without accidentally closing it completely.

In Fig. 4(d) a SEM image of nanotweezers with a $350 \mathrm{~nm}$ gap is shown. To fine-tune the gap and the lengths of the tips, parallel secondary tips are added to the converging primary tips. The parallel secondary tips are designed to grab elongated objects such as nanowires more effectively, which is difficult with sharp opposing tips. The final adjustments are done with deposition times of about 5 seconds, allowing the gap and tip lengths to be tuned to within $10 \mathrm{~nm}$, as shown in Fig. 4(e-f). With an acceleration voltage of 10 
$\mathrm{kV}$ and beam currents in the range 3-6 pA, we obtained a growth rate of up to $300 \mathrm{~nm} / \mathrm{min}$.

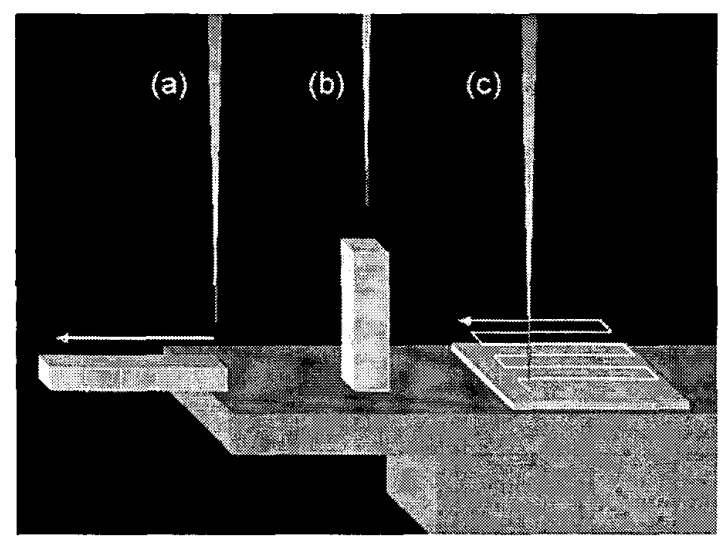

Figure 3. Electron beam induced growth modes. (a) Lateral growth. Movement of the electron beam will produce perpendicular or angled tips with respect to the surface. (b) Vertical growth: the electron beam is kept fixed. (c) Flood growth: carbon material is grown on an area by scanning the beam in a rectangular field.

An alternative and very useful method is lateral growth. Generally, the angle of the carbon tips with respect to the electron beam can be controlled by the lateral speed of the electron beam. By moving the electron beam with sufficient speed, the growth of the tip becomes perpendicular to the electron beam. The method is fast; using just the background contamination, a micron long tip with a diameter of 20 $40 \mathrm{~nm}$, can be grown in less than a minute. A set of nanotweezers fabricated this way is shown in Fig. 5 (top panel). While the vertical growth mode shown in Fig. 4 is more accurate, the lateral growth mode does not require any tilting and rotation of the sample stage and is consequently more suitable for automated fabrication. The requirements for such a system are, however, a mechanically stable SEM, a lithography system to control the beam position, and a very precise control of the growth rate, see for instance Ref. 12.

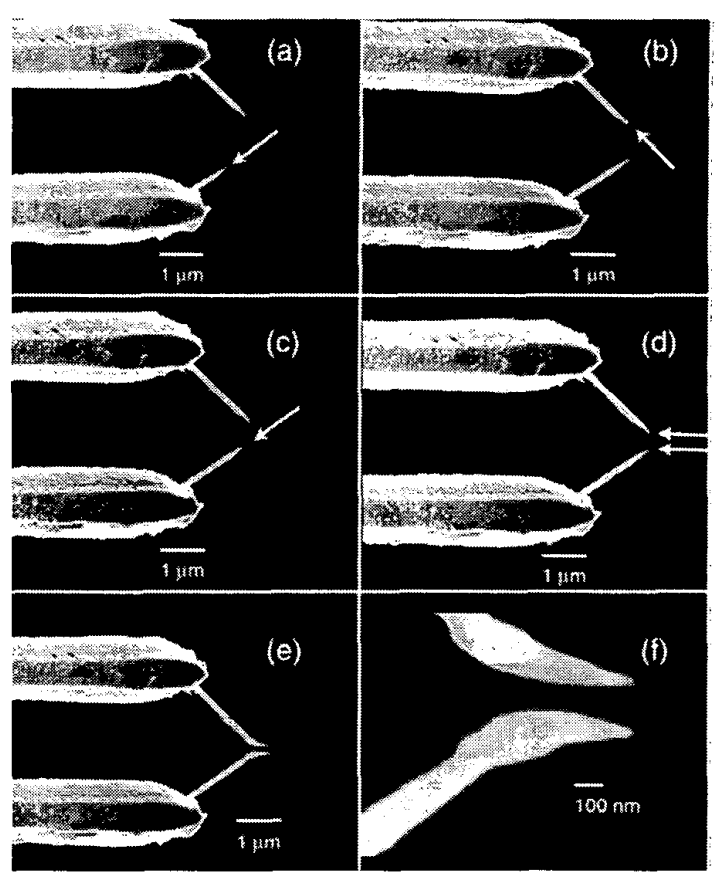

Figure 4. Nanotweezers made by vertical growth. The microactuator is tilted and rotated, to make the tips grow in converging directions. The arrows indicate the direction of the electron beam. Panels a-c show the step-by-step addition of tip elements until the gap is about $200 \mathrm{~nm}$. Panel d show's the addition of secondary tips, where the gap is tuned to $100 \mathrm{~nm}$. Panel e and $f$ show the finished tweezers at different magnifications.

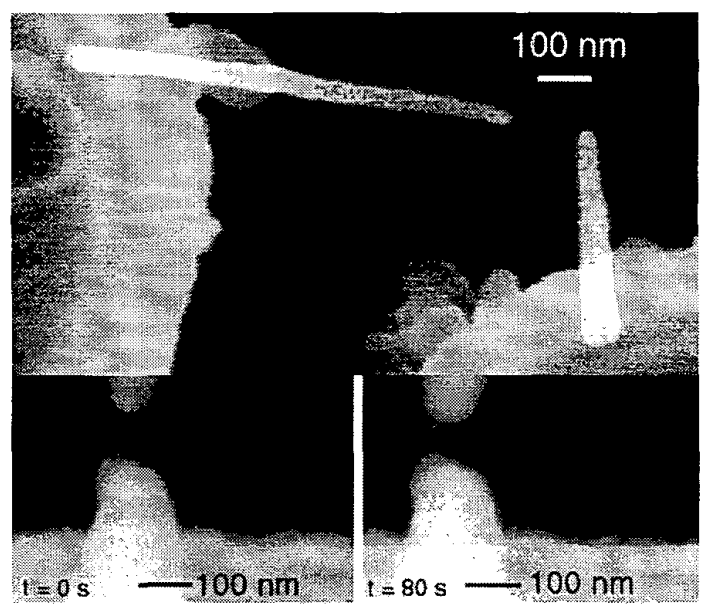

Figure 5. Top: two nanotips deposited by lateral growth, with a diameter of less than $50 \mathrm{~nm}$. The gap size is $130 \mathrm{~nm}$. Bottom: Flood growth. The gap size is decreased from $90 \mathrm{~nm}$ (left) to $45 \mathrm{~nm}$ (right) in 80 seconds while continuously monitoring the gap size. 


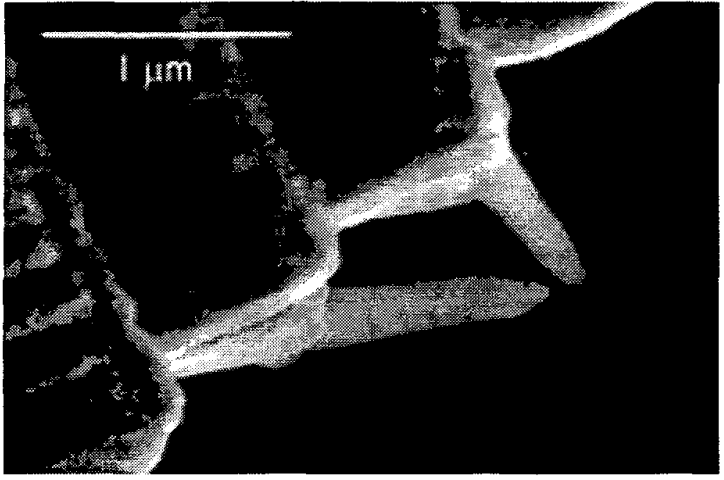

Figure 6. Nanotweezers with a $25 \mathrm{~nm}$ gap size. By a combination of vertical and flood growth modes a working nanotweezer device with a gap of just $25 \mathrm{~nm}$ was fabricated. The gap is imaged at the angle where the gap appears largest.

Both the vertical and the lateral growth modes make gap sizes of about $80-100 \mathrm{~nm}$ possible. To further decrease the gap, a flood growth is used. This is done simply by increasing the magnification so that only a small region containing the gap is imaged. The scanning of the electron beam induces an overall growth, decreasing the gap to the desired size, as seen in Fig. 5 (bottom panels). The set of nanotweezers exhibiting a $25 \mathrm{~nm}$ gap is shown in Fig. 6, was created by combination of the vertical and flood growth mode.

\section{Results}

\subsection{Actuation of nanotweezers}

For small deflections, Hooke's law combined with the fact that the electrostatic charge as well as the electric field increase proportionally with the applied voltage, will give rise to a roughly quadratic change in gap size as a function of voltage. The change in gap size was measured using an optical microscope, equipped with a CCD camera. By image analysis, the deflection could be measured to a few nanometers precision. Fig. 7 shows the deflection curves for opening and closing operation of the actuator. As expected, the measurement curve follows a parabola.

The actuation range, considered as the difference between the closed and open positions at a maximum voltage of $30 \mathrm{~V}$, was $335 \mathrm{~nm}$. Due to dielectric breakdown of the $1 \mu \mathrm{m}$ silicon oxide beams, higher voltages than $30 \mathrm{~V}$ are not feasible. Despite the extended gripper electrodes being more than $10 \mu \mathrm{m}$ from the driver electrodes (see inset of Fig. 7), the use of high actuation voltages increases the risk that stray electric fields may affect the manipulated object.

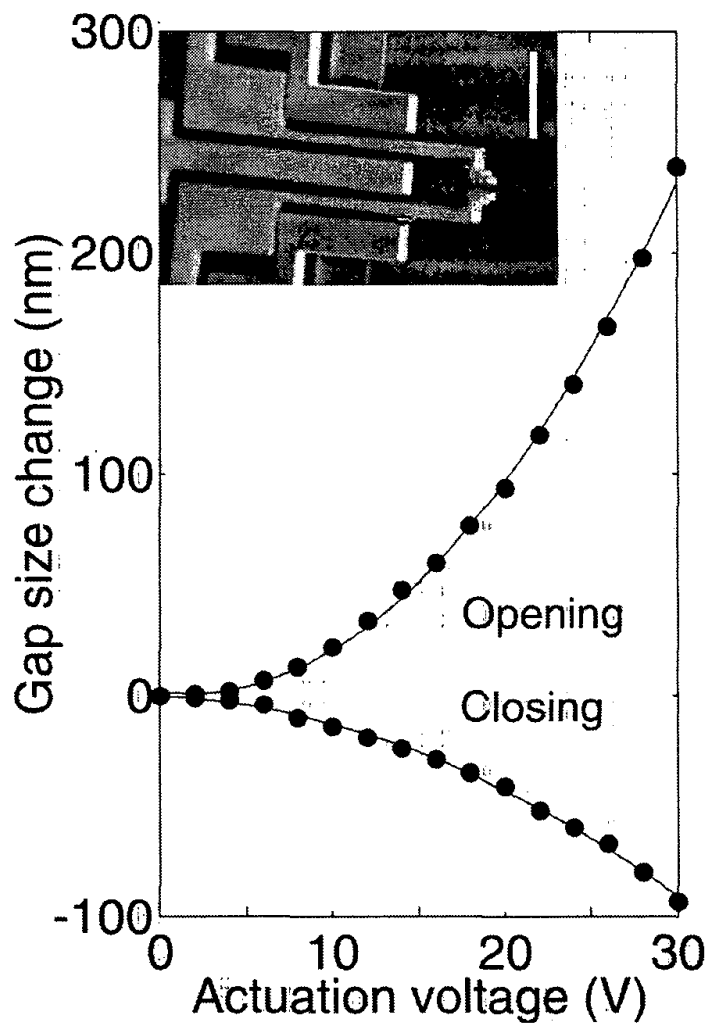

Figure 7. Change of gap size as a function of actuation voltage for a five-electrode microactuator. The actuator opens $240 \mathrm{~nm}$ and closes $95 \mathrm{~nm}$ by applying voltages of $30 \mathrm{~V}$. The full curves are parabolic fits to the data. The inset shows a SEM image of the device. The white bar is $10 \mathrm{um}$.

\subsection{Properties of nanotips}

A simple test of the mechanical and electrical properties of the metallized nanotips was performed using the optical microscope. A piece of silicon wafer covered with a thin layer of gold was positioned horizontally by a nanoresolution xyz-stage. The angle of the microelectrodes with respect to the sample surface was $30^{\circ}$ and that of the nanotips roughly $60^{\circ}$. By slowly lifting the sample towards the nanotips,. electrical contact was eventually achieved. An upper limit to the contact resistance of $1000 \Omega$ was established by two-point measurement. Sticking effects were observed upon retraction. The release point of the tip was 100-300 $\mathrm{nm}$ lower than the engage point, due to sticking of the gold-coated electrodes to the surface. The observed deflection of the cantilever corresponds to adhesion forces of the order of $10^{-6} \mathrm{~N}$.

Continued increase of the contact pressure eventually causes the nanotip to break, observed as a sudden increase of the measured contact resistance. 
Scanning electron microscope images confirmed that the nanotip and not the supporting microelectrode was broken. It was found that the tips typically broke at a contact force of about $10^{-5} \mathrm{~N}$, translating into a $1 \mu \mathrm{m}$ deflection of the cantilevers. The cantilevers themselves break at a deflection of 3-4 $\mu \mathrm{m}$. Despite their small dimensions, the nanoprobes are capable of deflecting the cantilevers to $25 \%$ of their maximal deflection. This supports the observation made in Ref. 11 , that carbon nanotips tend to deform elastically rather than break when exposed to rough treatment.

\subsection{Manipulation setup}

A setup for nanomanipulation in vacuum was built around a Klocke Nanoelectronics NMT xyz-translation stage. The $2 \mathrm{~nm}$ resolution xyz-stage was mounted inside a LEO 1500 scanning electron microscope. The setup is shown in Fig. 8(a). The angle and position of the sample can be set at will. A series of SEM images (Figs. 8(b-e)) shows a preliminary test of the setup. A tweezer tip is sliding over a latex bead with a diameter of $200 \mathrm{~nm}$, located on a gold surface. Due to charging of the latex beads by the electron beam, the beads adhere strongly to the surface. Although no manipulation was achieved, the experiment showed that the positional accuracy is better than $10 \mathrm{~nm}$, and that the imaging resolution of the microscope is of the order of $10 \mathrm{~nm}$. The setup provides sufficient imaging and positioning accuracy to manipulate sub-20 nm nanowires, which matches the minimal feature size of the nanotweezers.

\section{Perspectives}

Following the previously mentioned tests, the next natural step will be to position individual, free-standing nanowires, onto a set of electrodes. While the microstructure shown in the inset of Fig. 7 is designed as an actuator for the nanotweezers, it could also serve as a platform for testing nanowires and even for new devices. New devices can be built by attaching a nanowire between the ends of the gripper actuators. Such a device will be convenient for scanning across a surface. By changing the design slightly, the center driver electrode could be extended to perform as a gate electrode for the active component.

Force-feedback might be just as important for a two-finger device, as for single tip manipulation $[4,5]$. For a single tip, the laser beam reflected on an AFM cantilever, gives enough information to extract both vertical as well as lateral force components on the tip.

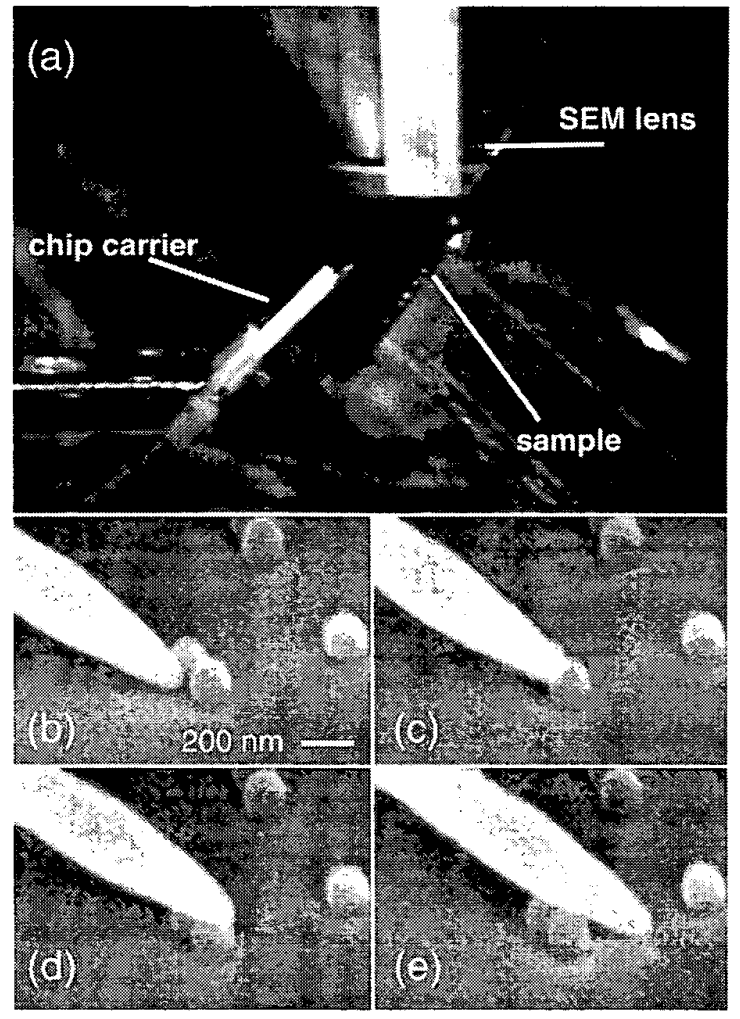

Figure 8. (a) Setup for nanomanipulation in a SEM. (b)-(e) a supertip is pushed towards a latex bead. The tip slides over the bead.

It is obviously more complicated to interpret the reflection from two independent submicron cantilevers, in a complex multi-cantilever system such as the one described here. A different method is capacitive readout, suggested by Ref. 17. By applying an AC voltage above the resonance frequency of the cantilevers, on top of the DC actuation voltage, the capacitance could be measured simultaneously. By comparing the actuation voltage with the actual electrode deflection, extracted from the measured capacitance, the applied force could be determined.

Providing successful implementation of forcefeedback in a suitable multi-finger manipulation system, a crude analogue to the human hand could be realized on the nanoscale. There are many problems to be solved, especially with regard to sticking of nanostructures to the nanotweezers. A method of in situ attachment of nanowires to surfaces is now being developed. Hence, we consider the customizable nanotweezers to have a potential of becoming a general-purpose tool for gripping, assembling and even sensing nanostructures.

Another interesting avenue for nanomanipulation is biological systems. We have developed a microfabrication process for a biocompatible version of 
the microactuator system, with titanium silicide cantilevers coated with a salt-water resistant nitride film. The nitride coating is opened at the ends of the microactuator. Submicron or nanoscale nanotweezers built on such actuators, might be used for electrical measurement or even manipulation of subcellular components such as ion channels in cell membranes. Several problems however have to be solved; how the electrostatic actuation will work in an ion-filled liquid, and how to monitor the nanotweezers while these are immersed in a liquid.

\section{Summary}

We have presented a novel type of nanotweezers with the unique feature of customizability. The shape of the tweezer tips can for the first time be adapted to suit the type of nanostructure of interest. The precise control of the design process allows us to tune the critical features to less than $10 \mathrm{~nm}$. The nanotweezers are based on an electrostatic microactuator with submicron features, providing individual control of the two tweezer electrodes.

Nanotweezers are a fairly new concept, which have yet to prove their usefulness by making new discoveries or new devices possible. We anticipate several routes by which the ongoing work presented here can eventually lead to a manipulation system that enables reliable and practical assembly of functional nanostructures.

\section{Acknowledgements}

We appreciate the technical assistance of I. Maximov and discussions with $\mathrm{C}$. Thelander and $\mathrm{M}$. Baller. We acknowledge financial support from the Danish Technical Research Foundation and the Swedish Strategic Research Foundation.

\section{References}

[1] D. M. Eigler and E. K. Schweizer, Positioning single atoms with a scanning tunneling microscope Nature 344: 524, 1990.

[2] T. Junno T, S-B Carlsson, Hongqi Xu, L. Montelius and L. Samuelson, Fabrication of quantum devices by Angstrom-level manipulation of nanoparticles with an atomic force microscope, Appl. Phys. Lett. 72: 548, 1998.

[3] U. Quaade, K. Stokbro, C. Thirstrup and F. Grey, Mechanism of single atom switch on silicon, Surf. Sci., 415: L1037-L1045, 1998.
[4] M. R. Falvo, R. M. Taylor, A. Helser, V. Chi, F. P. Brooks, S. Washburn and R. Superfine, Nanometre-scale rolling and sliding of carbon nanotubes, Nature, 397: 236, 1999.

[5] M. Setti and H. Hashimoto, Controlled Pushing of Nanoparticles: Modeling and Experiments, IEEE/ASME Transactions on Mechatronics, 5(2): 199-211, 2000.

[6] Ph. Avouris, T. Hertel, R. Martel, T. Schmidt, H. R. Shea, R. E. Walkup, Carbon nanotubes: nanomechanics, manipulation and electronic devices, Appl. Surf. Sc., 141: 201-209, 1999.

[7] M. Yu, M. J. Dyer, G. D. Skidmore, H. W. Rohrs, XueKun Lu, K. D. Ausman, J. R. Von Ehr and R. S. Ruoff, Three-dimensional manipulation of carbon nanotubes under a scanning electron microscope, Nanotechnology 10: 244-252, 1999.

[8] Yi Cui and C. M. Lieber, Functional Nanoscale Electronic Devices Assembled using Silicon Nanowire building blocks, Science, 291: 851-853, 2001.

[9] P. Kim and C. M. Lieber, Nanotube Nanotweezers, Science 386: 2148, 1999.

[10 K. Kakushima, M. Mita, D. Kobayashi, G. Hashiguchi, J. Endo, Y. Wada and H. Fujita, Micromachined tools for nano technology. Twin nano-probes and nano-scale gap control by integrated microactuators, IEEE MEMS 2001 Proc.p 294, 2001

[11] D. J. Keller, C. Chih-Chung, Imaging steep, high structures by scanning force microscopy with electron beam deposited tips, Surf. Sci. 268: 339, 1992.

[12 C. Schoessler $C$ and $H$. W. P. Koops, Nanostructured integrated electron source, J. Vac. Sci. Technol. B 16: 862, 1998.

[13] P. Bøggild, T. M. Hansen, O. Kuhn, F. Grey, T. Junno and L. Montelius, Scanning nanoscale fourpoint probes, Rev. Sci. Instr., 71: 2781, 2000.

[14]C. L. Petersen, T. M. Hansen, P. Bøggild, A. Boisen, O. Hansen and F. Grey, Scanning microscopic four-point conductivity probes, submitted to Sensors and Actuators.

[15]C.-J. Kim, A. P. Pisano, R. S. Muller, and M. G. Lim, Polysilicon Microgripper, Sensors and Actuators, A33:221-227, 1992.

[16]P. Bøggild, T. M. Hansen, C. Tanasa, F. Grey, Fabrication and actuation of customised nanotweezers with a 25 nanometer gap, Nanotechnology, in print.

[17] M. Baller, private communication. 